\title{
The genome of a wild Medicago species provides insights into the tolerant mechanisms of legume forage to environmental stress
}

\author{
Tianzuo Wang ${ }^{1}$, Lifei Ren', Caihong Li', Di Zhang ${ }^{1}$, Xiuxiu Zhang ${ }^{1}$, Gang Zhou², Dan Gao², Rujin Chen', \\ Yuhui Chen ${ }^{3}$, Zhaolan Wang ${ }^{4}$, Fengling Shi ${ }^{5}$, Andrew D. Farmer ${ }^{6}$, Yansu Li ${ }^{7}$, Mengyan Zhou ${ }^{*^{*}}$, \\ Nevin D. Young ${ }^{8}$ and Wen-Hao Zhang ${ }^{1,9^{*}}$ (D)
}

\begin{abstract}
Background: Medicago ruthenica, a wild and perennial legume forage widely distributed in semi-arid grasslands, is distinguished by its outstanding tolerance to environmental stress. It is a close relative of commonly cultivated forage of alfalfa (Medicago sativa). The high tolerance of $M$. ruthenica to environmental stress makes this species a valuable genetic resource for understanding and improving traits associated with tolerance to harsh environments.

Results: We sequenced and assembled genome of M. ruthenica using an integrated approach, including PacBio, Illumina, 10xGenomics, and Hi-C. The assembled genome was $904.13 \mathrm{Mb}$ with scaffold N50 of $99.39 \mathrm{Mb}$, and 50,162 protein-coding genes were annotated. Comparative genomics and transcriptomic analyses were used to elucidate mechanisms underlying its tolerance to environmental stress. The expanded FHY3/FAR1 family was identified to be involved in tolerance of $M$. ruthenica to drought stress. Many genes involved in tolerance to abiotic stress were retained in $M$. ruthenica compared to other cultivated Medicago species. Hundreds of candidate genes associated with drought tolerance were identified by analyzing variations in single nucleotide polymorphism using accessions of $M$. ruthenica with varying tolerance to drought. Transcriptomic data demonstrated the involvements of genes related to transcriptional regulation, stress response, and metabolic regulation in tolerance of $M$. ruthenica.
\end{abstract}

Conclusions: We present a high-quality genome assembly and identification of drought-related genes in the wild species of M. ruthenica, providing a valuable resource for genomic studies on perennial legume forages.

Keywords: Wild genetic resource, Genome, Evolution, Domestication, Comparative genomics, Transcriptome, Single-nucleotide polymorphism, Drought tolerance, Medicago ruthenica

\footnotetext{
* Correspondence: zhoumengyan@novogene.com; whzhang@ibcas.ac.cn

${ }^{2}$ Novogene Bioinformatics Institute, Beijing, China

${ }^{1}$ State Key Laboratory of Vegetation and Environmental Change, Institute of

Botany, The Chinese Academy of Sciences, Beijing, China

Full list of author information is available at the end of the article
}

(c) The Author(s). 2021 Open Access This article is licensed under a Creative Commons Attribution 4.0 International License, which permits use, sharing, adaptation, distribution and reproduction in any medium or format, as long as you give appropriate credit to the original author(s) and the source, provide a link to the Creative Commons licence, and indicate if changes were made. The images or other third party material in this article are included in the article's Creative Commons licence, unless indicated otherwise in a credit line to the material. If material is not included in the article's Creative Commons licence and your intended use is not permitted by statutory regulation or exceeds the permitted use, you will need to obtain permission directly from the copyright holder. To view a copy of this licence, visit http://creativecommons.org/licenses/by/4.0/ The Creative Commons Public Domain Dedication waiver (http://creativecommons.org/publicdomain/zero/1.0/) applies to the data made available in this article, unless otherwise stated in a credit line to the data. 


\section{Background}

Medicago ruthenica (L.) Trautv., an allogamous diploid $(2 n=2 x=16)$ perennial legume forage, is a native grassland species widely distributed in hillsides, embankments, mixed grass steppes, and meadows of Siberia, Mongolia, and northern China [1]. It is also a close relative of alfalfa ( $M$. sativa), which is the most important legume forage worldwide [2]. The distribution area of $M$. ruthenica is distinguished by dry, infertile soils, and long cold winter times [3]. Therefore, $M$. ruthenica must have evolved sophisticated mechanisms to survive the adverse environments, including drought, low temperatures, and infertile soil. Compared with other Medicago species, $M$. ruthenica is believed to be a relatively unique species among the Medicago species that are highly adapted to stressed environments, and whose potential application is positively evaluated in low-input systems, such as in the field without irrigation and in infertile soils [4].

Legume plants account for one third of primary crop yield as important sources for the consumption of human and animals in the world [5]. As the most important and popular legume forage, alfalfa has been cultivated in more than 80 countries with a total area of $\sim 32$ million hectares in the world [6]. Alfalfa is the top 10 crops in terms of cultivated area and is the third species only after soybeans and beans in legume crops (FAO, http://www.fao.org/faostat/en/\#data/QC). In the major areas across the world, alfalfa is frequently exposed to unfavorable growth conditions, such as drought in Argentina and northern China, low temperature in Russian and Canada, saline/alkaline soils in California of America and Australia. Alfalfa yield and quality have been negatively impacted by environmental stress in these areas [7-10]. The great attention paid to the traits associated with high yield during long-term domestication of cultivated alfalfa may render the cultivated alfalfa less tolerant to harsh environments. Therefore, genetic resources rich in genes tolerant to harsh environments are eagerly required for breeding alfalfa varieties with high tolerance to environmental stresses. Despite assembly of the genomes for several legume crops, only four legume forages, Lotus japonicas [11], M. truncatula [12], red clover (Trifolium pratense) [13], and alfalfa [14, 15], have been genome-sequenced so far. Red clover is perennial legume forage with mild tolerance to low temperature and drought [13]. L. japonicas and M. truncatula are annual legume species with limited agronomic application [11, 12]. The $M$. truncatula genome can only provide limited information for alfalfa because it differs in lifecycle and pollination from alfalfa [16]. The wholegenome of alfalfa was recently sequenced using cultivated cultivars $[14,15]$. However, the two alfalfa cultivars used for the whole-genome sequencing are of moderate tolerance to drought stress [17]. Numerous genes responsible for tolerance to environmental stress in the cultivated species may have been lost during domestication by selecting those traits associated with high yield and nutritional quality. Therefore, genomic information in the wild species can provide valuable clues for improving traits associated with adaptation to stressed environments in perennial legume forages.

As a close relative of alfalfa, $M$. ruthenica is also a perennial species with comparable genome size, life cycle, and pollination strategies to alfalfa. More importantly, as a wild species with many accessions occurring widely in the arid and/or semi-arid areas, it is highly tolerant to drought stress and has been used as parental materials to improve alfalfa tolerance to adverse environments by breeding alfalfa cultivars tolerant to environmental stress $[2,18]$. Therefore, $M$. ruthenica can be a valuable model forge to study molecular mechanisms underlying tolerance to environmental stress in legume forages in general and alfalfa in particular. Moreover, comparative genomes of cultivated alfalfa with their wild relatives can identify the useful alleles in the wild species for trait improvement of cultivated alfalfa, thus contributing to our understanding of cultivated alfalfa's domestication process. However, genomic information of M. ruthenica is scarce, which greatly hampers our molecular elucidation of stress physiology and its application to genomic improvements in tolerance of alfalfa to environmental stress. To facilitate genomic improvements of alfalfa in terms of enhanced tolerance to abiotic stress and understand the mechanisms underlying the great tolerance of $M$. ruthenica to environmental stress, we assembled and analyzed the genome of $M$. ruthenica that occurs naturally in arid and semi-arid areas of northern China. We further explored the mechanisms underlying the super tolerance of $M$. ruthenica to drought stress by an integrated approach. We firstly compared the transcription factor genes of $M$. ruthenica with M. truncatula and $M$. sativa. We further analyzed single-nucleotide polymorphism (SNP) variations and identified hundreds of candidate genes associated with drought tolerance using 20 accessions of $M$. ruthenica collected across different geographic sites in China. Numerous drought-responsive genes were identified by transcriptome, and their roles in the regulation of drought tolerance in the $M$. ruthenica were discussed.

\section{Results}

\section{Genome sequencing and assembly}

By K-mer analysis, the genome size and heterozygosity of $M$. ruthenica (Xinghe accession) were estimated to be about $914 \mathrm{Mb}$ and $2.2 \%$, respectively (Additional file 1 : Figure S1, Additional file 2: Table S1). The genome was bigger than monoploid genome of cultivated alfalfa 
$(\sim 800 \mathrm{Mb})[14,15]$ and much larger than that of $M$. truncatula $(\sim 500 \mathrm{Mb})$ [12]. We assembled $96.32 \mathrm{~Gb}$ PacBio single-molecule long reads $(105 \times)$ and $301.44 \mathrm{~Gb}$ Illumina shotgun reads $(330 \times)$ by sequencing paired-end and mate-pair libraries (250 bp-20 Kb). Moreover, 119.3 Gb data $(131 \times)$ were obtained from the linked readsequencing library by $10 \times$ Genomics platform to assist assembly (Additional file 2: Table S2). Using these data, we generated a Version 0.8 genome with the contig N50 size of $632.05 \mathrm{~Kb}$ and scaffold N50 size of $2.17 \mathrm{Mb}$ mainly by the FALCON algorithm (Additional file 2: Table S3). To anchor the scaffolds to the chromosomes, 106.45 Gb high-throughput chromosome conformation capture $(\mathrm{Hi}-\mathrm{C})$ data $(116 \times)$ were mapped to the Version 0.8 genome (Additional file 1: Figure S2). A total of 825.32 $\mathrm{Mb}$ representing $91.3 \%$ of the total assembled genome length was anchored to the eight chromosomes of $M$. ruthenica with the length of 90.05 120.35 Mb (Fig. 1a). The Version 1.0 genome was generated with the scaffold N50 size of $99.39 \mathrm{Mb}$, and GC proportion of 35.9\% using LACHESIS (Table 1, Fig. 1b, Additional file 1: Figure S3, Additional file 2: Table S4). The completeness of genome assembly was evaluated by calculating the genome coverage rate $(99.8 \%)$ to our assembled genome. The mapping rate of the Illumina paired-end reads and PacBio reads was $93.7 \%$ and $97.9 \%$, respectively. Transcriptome data were also used to evaluate the completeness of genome assembly. The average $93.2 \%$ of transcriptomic reads was accurately mapped to the final genome assembly (Additional file 2: Table S5). The transcriptome assembly composing of 45,397 unigenes ( $>500$ bp) was mapped to the genome assembly. More than $96.5 \%$ of these unigenes were identified in the $M$. ruthenica genome and $92.8 \%$ of them were covered more than $50 \%$ by one scaffold (Additional file 2: Table S6). Moreover, BUSCO analysis revealed that $91.3 \%$ of 1375 single-copy ortholog genes from the Embryophyta were complete in the genome assembly $(83.2 \%$ as single-copy, $8.1 \%$ as duplicates). Conserved Core Eukaryotic Gene Mapping Approach (CEGMA) analysis revealed that $94.0 \%$ of the core protein-coding genes were identified in our assembled genome. These results suggest that the highly complete genome of $M$. ruthenica is assembled in the present study.

\section{Genome annotation}

Repetitive sequences comprised $57.0 \%$ in the genome of $M$. ruthenica, including the tandem repeat sequences

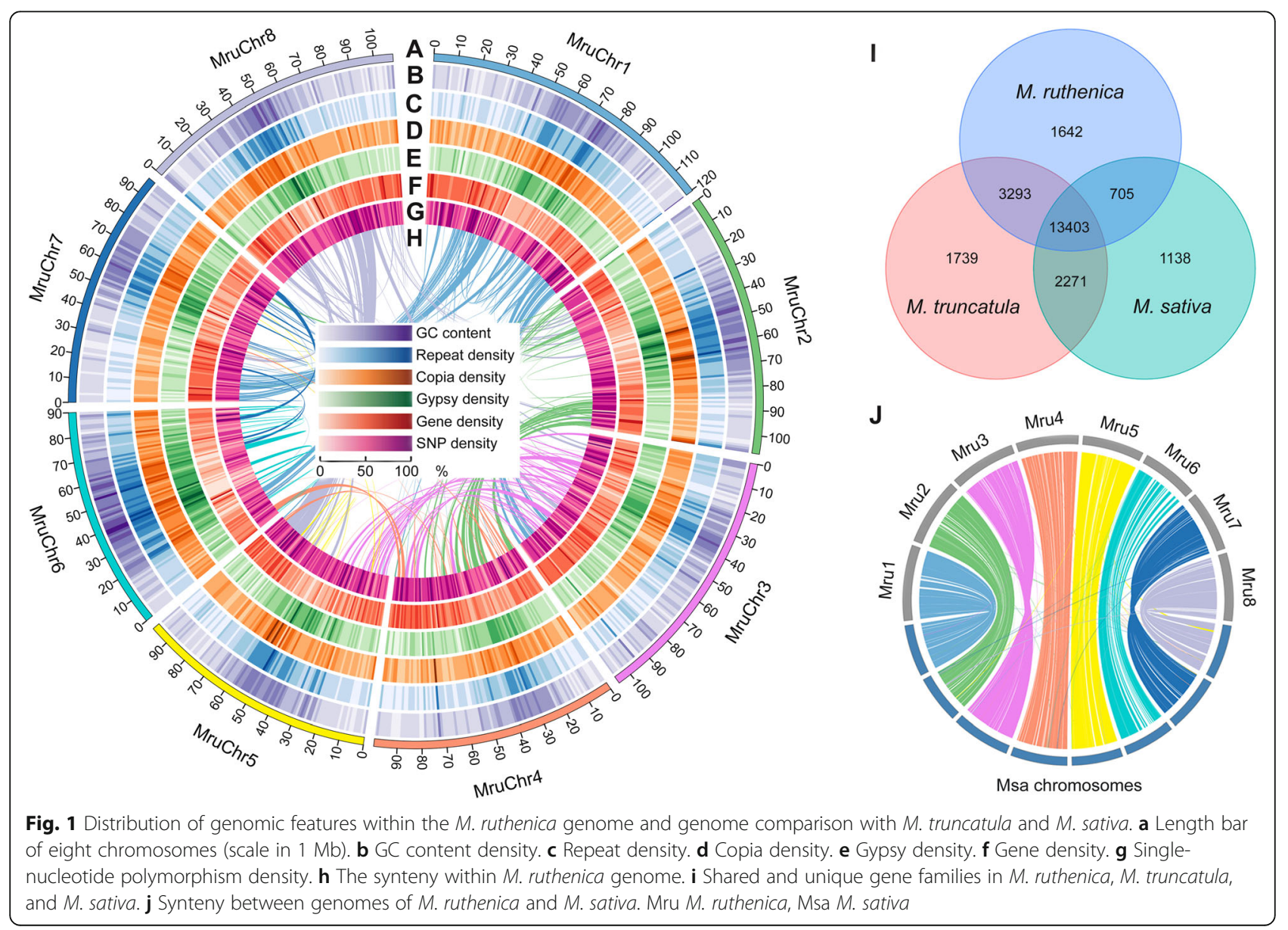


Table 1 The statistics of the M. ruthenica genome

\begin{tabular}{|c|c|c|c|c|}
\hline \multirow[t]{3}{*}{ Genome assembly } & \multirow{2}{*}{ Contigs } & \multirow{2}{*}{$\begin{array}{l}\text { N50 } \\
612.99 \mathrm{~Kb}\end{array}$} & \multirow{2}{*}{$\begin{array}{l}\text { N90 } \\
247.80 \mathrm{~Kb}\end{array}$} & \multirow{3}{*}{$\begin{array}{l}\text { Total length } \\
902.93 \mathrm{Mb} \\
904.13 \mathrm{Mb}\end{array}$} \\
\hline & & & & \\
\hline & Scaffolds & $99.39 \mathrm{Mb}$ & $90.05 \mathrm{Mb}$ & \\
\hline \multirow[t]{13}{*}{ Genome annotation } & Repetitive sequences & Categories & Length (bp) & Percentage (\%) \\
\hline & & DNA transposon & $24,361,896$ & 2.6841 \\
\hline & & LTR & $470,694,297$ & 51.859 \\
\hline & & LINE & $15,815,260$ & 1.7425 \\
\hline & & SINE & 81,673 & 0.0090 \\
\hline & & Other & $6,173,813$ & 0.6753 \\
\hline & Protein-coding genes & Number & Annotated number & Average CDS length \\
\hline & & 50,162 & 49,176 & $1050 \mathrm{bp}$ \\
\hline & Non-coding RNAs & Categories & Number & Percentage (\%) \\
\hline & & miRNA & 3820 & 0.0538 \\
\hline & & tRNA & 788 & 0.0065 \\
\hline & & rRNA & 364 & 0.0050 \\
\hline & & snRNA & 2892 & 0.0355 \\
\hline
\end{tabular}

LTR long terminal repeat, LINE long interspersed nuclear elements, SINE short interspersed nuclear elements, CDS coding sequence

and transposable elements (Fig. 1c, Additional file 2: Table S7). Long terminal repeat (LTR) retrotransposons were the most abundant transposable elements, comprising $51.9 \%$ of the whole genome, including Copia elements $(21.0 \%$ of genome) and gypsy elements $(27.1 \%$ of genome) (Fig. 1d, e, Additional file 1: Figure S4, Additional file 2: Table S8). In soybean, common bean, pigeonpea, and alfalfa genomes, LTR retrotransposons were also the most abundant transposable elements, representing $42.0 \%, 36.7 \%, 37.1 \%$, and $27.4 \%$ of their genome size, respectively [19-21].

To predict protein-coding genes, we sequenced transcriptomes from roots, stems, leaves, flowers, and pods. In addition, we performed de novo and homolog-based predictions. The final reference gene set contained 50, 162 protein-coding genes, with $3339 \mathrm{bp}$ transcripts, 4.02 exons, and 1050 bp coding sequences on average (Fig. 1f, Additional file 1: Figure S5, Additional file 2: Table S9). The density of genes was correlated with that of single-nucleotide polymorphism (SNP) in some positions in chromosomes (Fig. 1g).

Of all the protein-coding genes in M. ruthenica, 49, 176 genes (accounting for 98.0\%) were annotated (Additional file 2: Table S10). The number of protein-coding genes in $M$. ruthenica genome was similar with that in $M$. truncatula and $M$. sativa $[12,15]$, but it was more than that in chickpea [22] and red clover [13] (Additional file 2: Table S11). After clustering, 27,764 gene families and 843 common single-copy orthologs were detected across $M$. ruthenica and six other species by OrthoMCL (Additional file 1: Figure S6). Of them, 13, 403 gene families were identified as common among the three Medicago species (Fig. 1i). We further identified unique genes from the $M$. ruthenica genome, and their Gene Ontology (GO) terms were analyzed (Additional file 2: Table S12). Multiple enriched GO terms related to stress response were identified, including cellular response to stimulus (GO: 0051716), response to stress (GO: 0006950), response to stimulus (GO: 0050896), and response to wounding (GO: 0009611). In addition, two GO terms related to DNA replication (GO: 0006281, DNA repair; GO: 0006260, DNA replication) were enriched. These enriched GO terms suggest that there is a specific mechanism to resist stresses and repair DNA after damage, which may contribute to the tolerance of $M$. ruthenica to stresses. Moreover, we predicted 3820 microRNAs, 2892 snRNAs, 788 tRNAs, and 364 rRNAs in the $M$. ruthenica genome (Additional file 2: Table S13).

The genome size of $M$. ruthenica was about two times greater than that of $M$. truncatula. The repetitive sequence length in the genome of $M$. ruthenica was much longer than that of M. truncatula (517 Mb vs. $125 \mathrm{Mb}$ ) [12]. This may explain the greater genome size of $M$. ruthenica than that of $M$. truncatula. We further compared the density of transposable elements along the eight chromosomes between the two species and found that they were much more abundant in the genome of $M$. ruthenica (Additional file 1: Figure S7). Moreover, despite similar number of protein-coding genes in the two species (Table 1, Additional file 2: Table S11), the average length of transcripts in the $M$. ruthenica genome was longer than that in M. truncatula (3339 bp vs. 2332 bp). 


\section{Genome evolution and synteny analysis}

The abovementioned 843 common single-copy orthologs were used to construct the phylogenetic tree by RAxML software. In the Papilionoideae subfamily, the galegoid (M. ruthenica, M. sativa, $M$. truncatula, C. arietinum, and L. japonicus) and millettioid (G. max) clades separated $\sim 46.5$ millions of years ago (Mya). Within the galegoid clade, the split of $M$. ruthenica was estimated at $\sim 8.5$ Mya from M. truncatula and M. sativa, and the divergence from red clover and chickpea was estimated at $\sim 19.1$ Mya and at $\sim 28.2$ Mya, respectively (Fig. 2a).

We further constructed the genome synteny within and among $M$. ruthenica, M. truncatula, chickpea, and red clover by Mcscan software, respectively (Fig. 1h, Additional file 1: Figure S8, Additional file 1: Figure S9, Additional file 1: Figure S10, Additional file 2: Table S14). Most chromosomes in the $M$. ruthenica genome aligned mainly with their corresponding $M$. truncatula chromosomes. However, the chromosome 4 and 8 of $M$. truncatula exhibited translocation by analyzing its genome synteny with $M$. ruthenica and chickpea, suggesting that the chromosome rearrangements in the genome of M. truncatula have occurred after speciation (Additional file 2: Table S15). We also analyzed the genome synteny between $M$. ruthenica and $M$. sativa using the genome data of the most recent genome of alfalfa and cultivated alfalfa at the diploid level (Fig. 1j, Additional file 1: Figure S11). These results suggest that the two legume species are closely related in terms of genome sequence.

We extracted all the duplicated gene pairs from the syntenic blocks and calculated the four-fold degenerate third-codon transversion (4DTv) distance. M. ruthenica, $M$. truncatula, and $M$. sativa shared a whole genome duplication event (4DTv 0.32). The 4DTv distance of $M$. ruthenica-M. truncatula orthologs and $M$. ruthenica$M$. sativa orthologs confirmed that the three species share a genome duplication event (Fig. 2b).

\section{Transcription factors and gene family expansion/ contraction analysis}

We identified $2402 \mathrm{TFs}$ in $M$. ruthenica genome by comparing their sequences with the known domains of TFs using iTAK. The number of TFs accounted for $4.8 \%$ of the 50,162 protein-coding genes, and the TFs were distributed in 49 families. In addition, we identified 2207 TFs that belong to 49 families in the $M$. truncatula genome, and $2638 \mathrm{TFs}$ that belong to 73 families in the $M$. sativa genome (Fig. 3a, Additional file 2: Table S16). The three species shared 47 common TF families. Farred elongated hypocotyl 3/Far-red impaired response 1 family (FHY3/FAR1) is a positive transcription factor in the phytochrome A pathway. We found 291 FHY3/FAR1 genes in the genome of $M$. ruthenica, which was the largest TF family in M. ruthenica. We checked the authenticity for all the 291 FHY3/FAR1 genes one by one using Pacbio long reads by the IGV software and found all these genes were covered by Pacbio long reads. And the FPKM value (fragments per kilobase of exon model per million mapped fragments) of 175 FHY3/FAR1 genes was more than 0.1 (Additional file 2: Table S17). The TF number in the most common families of the three species was comparable, and members of the FHY3/FAR1 genes in $M$. ruthenica were greater than those in $M$. truncatula and M. sativa by 220 and 126, respectively. Phylogenetic tree of FHY3/FAR1 family in the three Medicago species showed expansion of this family in the $M$. ruthenica genome originated from several paralogs of FHY3/FAR1 family (Fig. 3b). We found a significant cluster of $M$. ruthenica FHY3/FAR1 family in the phylogenetic tree, which consisted of 69 M. ruthenica branches, 13 M. truncatula branches, and 1 M. sativa branch. Moreover, 122 FHY3/FAR1 genes occurred in the synteny blocks across the eight chromosomes in the M. ruthenica genome, and two genes (FHY3/FAR1-200 and FHY3/FAR1-201) occurred in clusters, suggesting
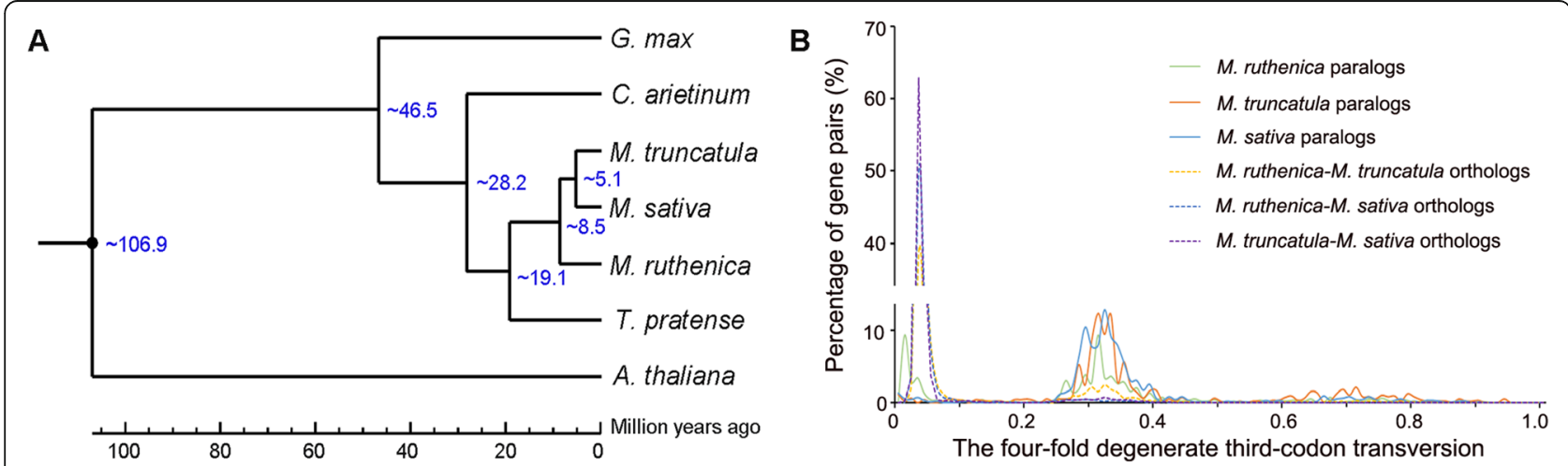

Fig. 2 Phylogenetic tree, divergence time of seven species (a), and the four-fold degenerate third-codon transversion rate of gene pairs in three Medicago species (b). The phylogenetic tree was constructed based on 843 single-copy orthologous genes across $M$. ruthenica and the other six species by RAxML software, and the divergence times (Mya) are indicated by the blue numbers beside the branch nodes in panel $\mathbf{a}$. The common whole-genome duplication events are indicated by the peaks ( $4 \mathrm{DTV}=0.32$ ) in panel $\mathbf{b}$ 


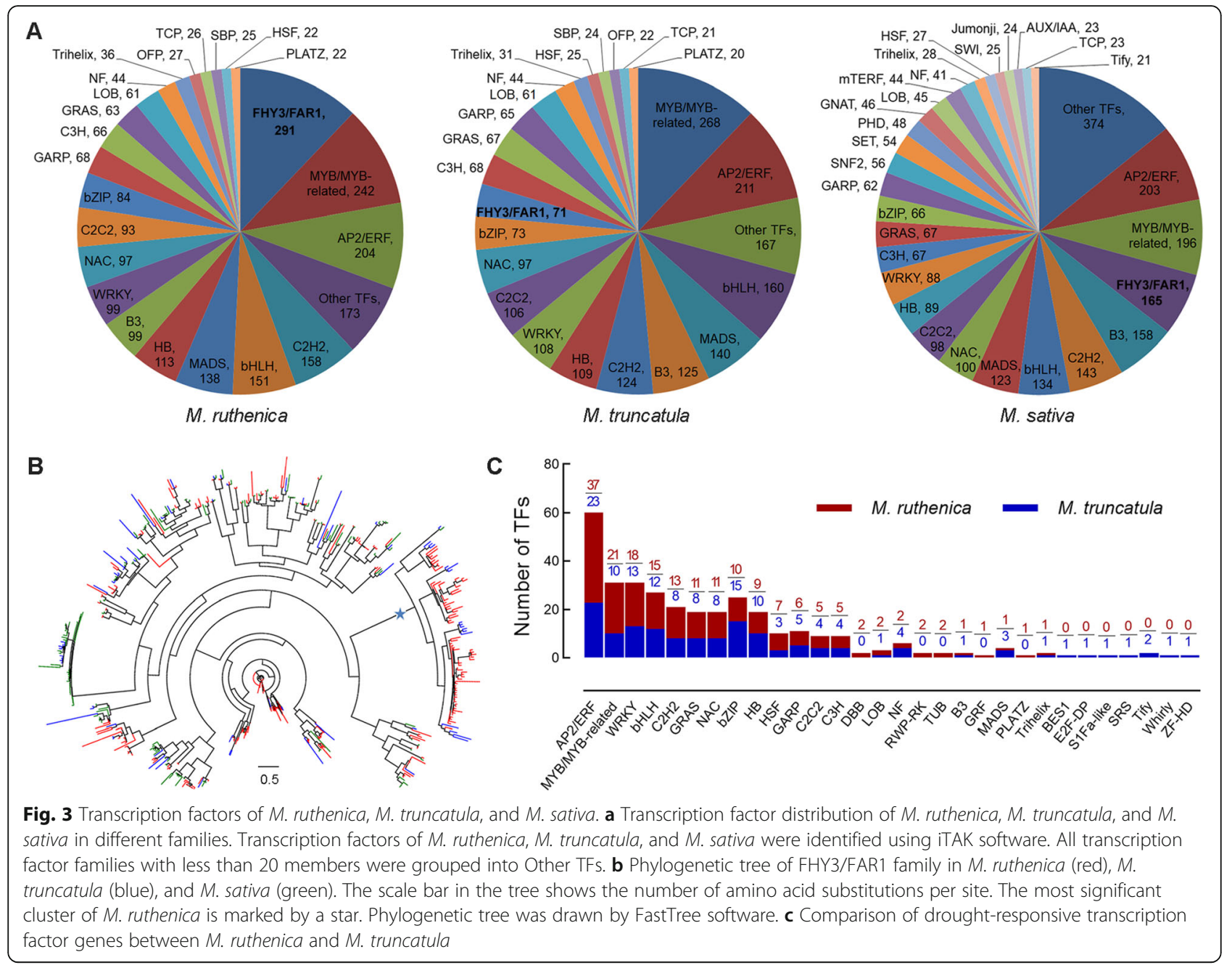

that MruFHY3/FAR1 genes originate from both whole genome duplication and tandem duplication (Additional file 1: Figure S12; Additional file 2: Table S17). In contrast, no $F H Y 3 / F A R 1$ genes were identified in clusters of the genome of $M$. truncatula, suggesting that MtrFHY3/ FAR1 genes only originate from whole genome duplication (Additional file 2: Table S18).

We also analyzed other expanded and contracted gene families (Additional file 2: Table S19, Additional file 2: Table S20). GO analysis of expanded families led to the enriched terms that were related to stress response (GO: 0006950, response to stress), signal transduction (GO: 0004965, G-protein-coupled GABA receptor activity), mineral element absorption (GO: 0008272, sulfate transport; GO: 0030955, potassium ion binding), and regulation of gene expression (GO: 0006306, DNA methylation; GO: 0003676, nucleic acid binding).

\section{Analysis of genome variation during domestication}

The genome of $M$. ruthenica was used as a reference to analyze the genome variation of $M$. truncatula and $M$. sativa during domestication. A total of 1269 and 309 genes were generated in $M$. truncatula and $M$. sativa during domestication, respectively. Further, we found that 1954 and 579 genes were lost in M. truncatula and $M$. sativa during domestication, respectively. A total of 45 and 22 GO terms were enriched based on these lost genes in M. truncatula and M. sativa, respectively (Additional file 2: Table S21, Additional file 2: Table S22). Some GO terms related with stress tolerance were enriched for M. truncatula and M. sativa, such as cellular response to stimulus (GO: 0051716), nucleic acid binding (GO: 0003676), and DNA repair (GO: 0006281).

\section{Comparison of drought tolerance among legume forages}

The wide occurrence of $M$. ruthenica in the arid and semi-arid areas prompts us to evaluate the molecular characteristics underlying its tolerance to drought. We compared the drought tolerance of $M$. ruthenica with that of T. pretense, M. truncatula, M. varia, M. falcata, and two alfalfa cultivars. Seedlings of $M$. ruthenica exhibited greatest tolerance to drought stress among the 
legume species examined (Additional file 1: Figure S13). For example, exposure of seedlings to drought led to marked reductions in survival rates for other legume forages, while the same treatment had little effect on survival rate of $M$. ruthenica seedlings, highlighting the strongest tolerance of $M$. ruthenica to drought stress among the legume forages examined. Previous studies have shown that the drought tolerance of $M$. varia and $M$. falcata was greater than the two alfalfa cultivars used for genome-sequencing [17]. Therefore, the drought tolerance of $M$. ruthenica is much stronger than that of the two alfalfa cultivars.

To identify the potentially key genes underlying the drought tolerance, root samples of genome-sequenced $M$. ruthenica (Xinghe accession) exposed to drought stress for varying times were used to construct cDNA libraries. High-throughput sequencing (RNA-seq) of 12 libraries led to generation of $84.90 \mathrm{G}$ clean data (Additional file 2: Table S23). Drought-responsive TF genes were identified by comparing the normalized expression levels of genes between libraries of drought stress and control using DESeq. We identified $183 \mathrm{TF}$ genes that were responsive to drought at the 7th day of drought treatment (Additional file 2: Table S24), while 144 drought-responsive TF genes were detected in $M$. truncatula under the identical drought regime (Fig. 3c, Additional file 2: Table S25). Furthermore, we found that drought-responsive members of AP2/ERF family were the most abundant in both species. For example, we detected 37 and 23 drought-responsive TF genes of AP2/ERF family in $M$. ruthenica and $M$. truncatula, respectively. Of MYB/MYB-related family, we identified 21 and 10 drought-responsive TFs in $M$. ruthenica and $M$. truncatula, respectively. In addition, several droughtresponsive TF families, including WRKY, bHLH, C2H2, GRAS, NAC, bZIP, and HB, were markedly regulated in the two species under drought stress (Fig. 3c).

\section{Selective sweep analysis among different accessions of $M$. ruthenica}

Given the wide distribution of $M$. ruthenica in China [23], we compared tolerance of different accessions to drought stress by collecting seeds of $M$. ruthenica 20 accessions from different locations across northern China (Fig. 4a, b). We found significant correlations between the survival rates of the $20 \mathrm{M}$. ruthenica accessions under the drought stress and annual precipitation at sites where their seeds originally collected (Fig. 4c). This result suggests that tolerance to drought may result from evolutionary adaptation of $M$. ruthenica to the arid environments. Of the $20 \mathrm{M}$. ruthenica accessions, the Zhenglanqi accession exhibited the highest survival rate under drought stress (Fig. 4b, d).
To identify single-nucleotide polymorphism (SNP) variation associated with drought tolerance among different $M$. ruthenica accessions, we performed whole genome resequencing of 19 accessions in addition to Xinghe accession that was used for the whole-genome sequencing. We obtained a total of 222.84 G clean data, and their 13x average depth for the 19 accessions (Additional file 2: Table S26). We obtained a total of 14,212,747 SNPs with high quality (Additional file 2: Table S27). A rooted phylogenetic tree was constructed using the SNP information by neighbor-joining method (Fig. 4e), and principal component and population structure were also analyzed (Additional file 1: Figure S14; Additional file 1: Figure S15). In addition, according to the survival rates of the 20 accessions under drought stress (Fig. 4b), we grouped the top 7 tolerant accessions as the tolerant population, while the least 7 tolerant accessions were set as the control population. The population differentiation (Fst) and nucleotide diversity ratio $\log _{2}\left(\theta \pi_{\text {Control }} / \theta \pi_{\text {Tolerance }}\right)$ were calculated using SNP data across the $40 \mathrm{~kb}$ windows with a $20 \mathrm{~kb}$ slide (Fig. 4f, g, Additional file 1: Figure S16). These allowed us to identify 367 candidate genes associated with drought tolerance (Additional file 2: Table S28). GO analysis led to enriched terms related with photosynthesis (GO: 0019684, photosynthesis, light reaction; GO: 0022900, electron transport chain; GO: 0003843, 1,3- $\beta$-Dglucan synthase activity) and stress response (GO: 0006979, response to oxidative stress; GO: 0004601, peroxidase activity) (Additional file 2: Table S29).

\section{Transcriptome analysis}

To explore the regulatory mechanisms of gene expression under drought stress, the most drought tolerant Zhenglanqi accession was selected to perform transcriptome analysis, with the Xinghe accession whose genome had been assembled in this study as control. Root samples treated with drought stress of varying times were used to construct cDNA libraries. High-throughput sequencing (RNA-seq) of 24 libraries led to generation of 168.29 G clean data (Additional file 2: Table S23). Drought-responsive genes were identified by comparing the normalized expression levels of genes from drought stress and control libraries using DESeq. Exposure to drought stress led to alterations in expression patterns of 1383 and 1693 genes in roots of the accessions collected from sites of Zhenglanqi and Xinghe, respectively. The number of drought-responsive genes peaked at the 7th day among different treatments of stringency (Additional file 1: Figure S17). A network of drought-responsive genes was constructed using STRING (Additional file 1: Figure S18), and the GO terms of drought-responsive genes were enriched (Additional file 2: Table S30, Additional file 2: Table S31). Taken the 7 th day of drought stress as an 


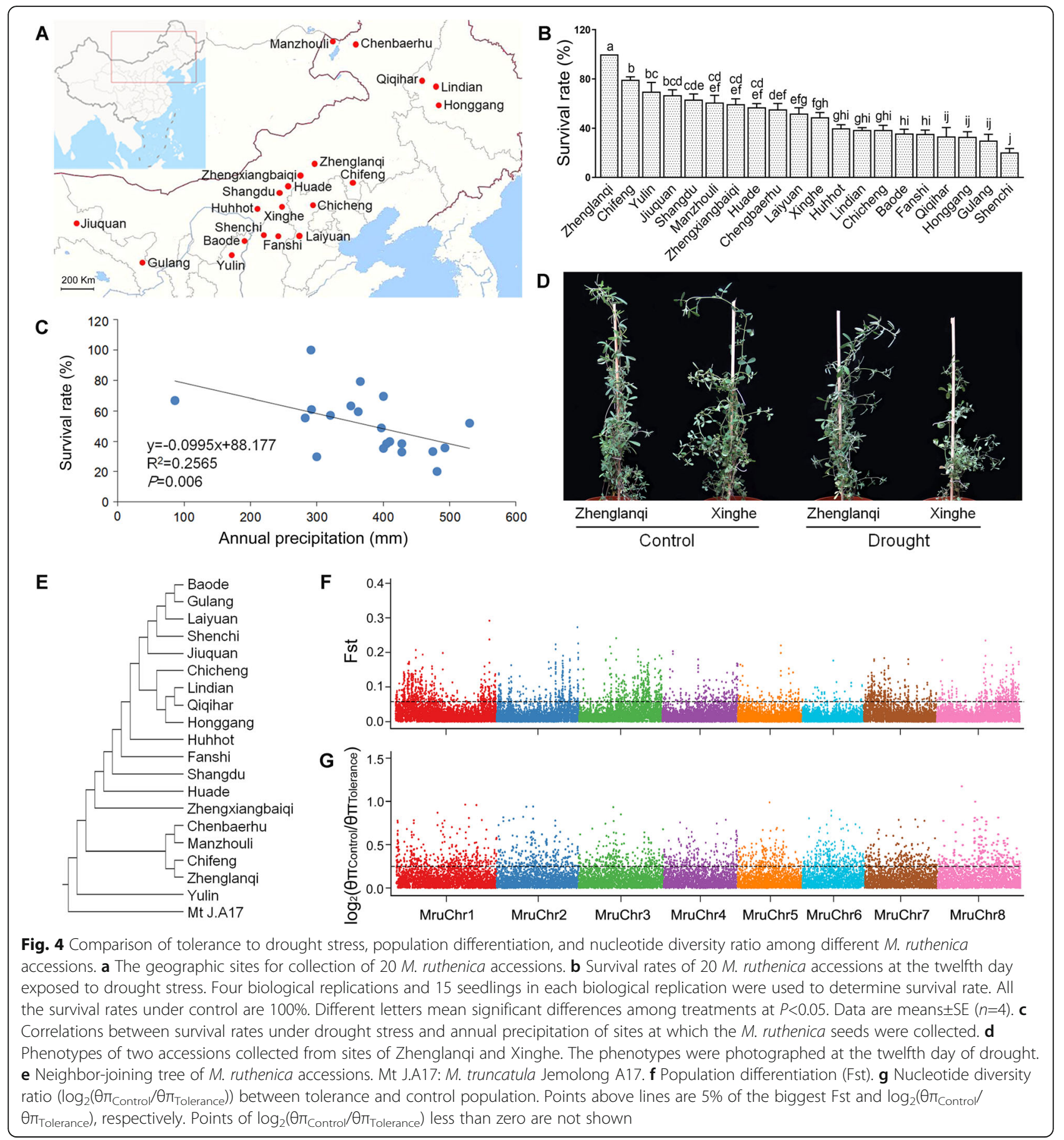

example, we found that the Zhenglanqi accession exhibited greater enrichments of GO terms compared to Xinghe accession (79 vs. $30 \mathrm{GO}$ terms) and that the GO terms in molecular functions (GO:0016209, antioxidant activity; GO:0001071, nucleic acid binding transcription factor activity) and biological processes (GO:0006979, response to oxidative stress) were enriched in the two accessions (Fig. 5).

\section{Discussion}

Medicago ruthenica is a wild legume forage with super tolerant to varying abiotic stresses. Therefore, it provides a valuable genetic resource for improving traits associated with tolerance to environmental stress in legume forage in general and in alfalfa in particular [3, 24]. However, the lack of reference genome for the perennial legumes with super tolerance greatly hampers our 


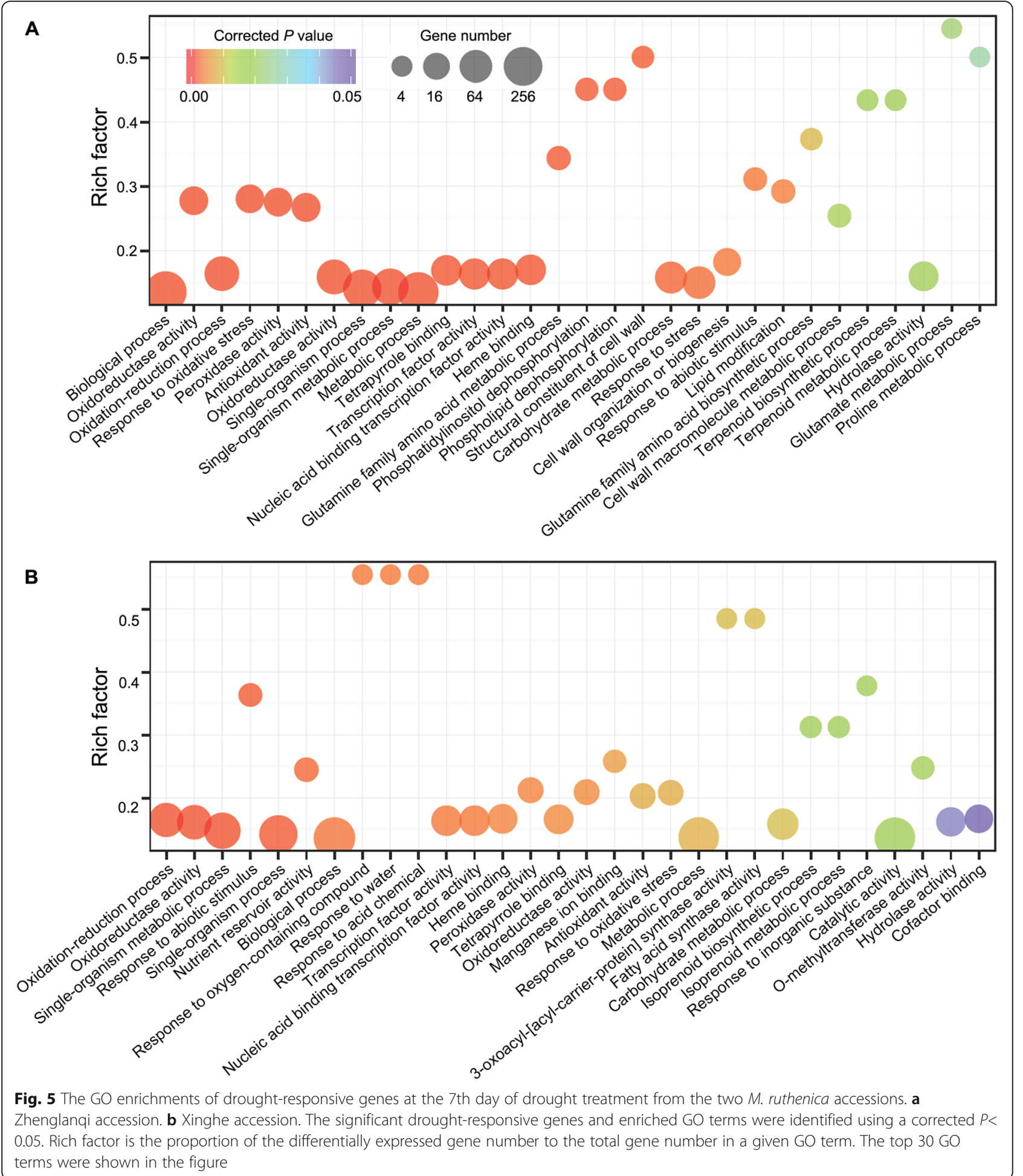

molecular understanding how perennial legume forages respond and adapt to the harsh environments. To fill this gap, we assembled a chromosome-scale genome of $M$. ruthenica in high quality using $623.51 \mathrm{~Gb}$ data (682x of genome size). To the best of our knowledge, our genome sequence is the first one for a wild legume forage species with great tolerance to environment stress.

The ancestors of all flowering plants have experienced the whole genome duplication (WGD), which is an important evolutionary force for speciation, adaptation, and diversification $[25,26]$. Moreover, the WGD event 
often occurs in the ancestors of the species-rich groups, including legumes [27]. The analysis of M. truncatula, G. max, and other Papilionoid genomes of legumes characterized a common WGD event in the Papilionoideae subfamily $[12,20]$. To unravel the genome evolution, we constructed the genome synteny among $M$. ruthenica, M. truncatula, and alfalfa (Fig. 1i, Additional file 1: Figure S8). The analysis of 4DTv distance revealed that the three species shared the ancestral Papilionoideae WGD (Fig. 2b). We identified 2977 orthologous genes between $M$. ruthenica and $M$. sativa from their own paralogous blocks (Additional file 1: Figure S19). This result illustrates that $M$. ruthenica and $M$. sativa shares the ancestral Papilionoideae WGD and have undergone a similar evolutionary course. The left genes present in the paralogous blocks were retained in the different species during genome recombination after the WGD or generated after speciation. These genes may contribute to the origin of species specificity.

The gene family expansion can enhance adaptations to changing environments [28]. FHY3/FAR1 family in $M$. ruthenica was the largest among those in the galegoid clades of Papilionoideae subfamily according to the known genome information. FHY3/FAR1 family has been suggested to directly activate the expression of $H E M B 1$ and $A B I 5$, which regulates chlorophyll biosynthesis [29], and ABA-dependent tolerance to abiotic stress [30], respectively (Fig. 6, Additional file 1: Figure S20A). Furthermore, FHY3/FAR1 can detoxify ROS by upregulating expression of MIPS1 to enhance the biosynthesis of inositol [31]. This may explain the higher expression of MrMIPS1 and less accumulation of in $\mathrm{H}_{2} \mathrm{O}_{2}$ in $M$. ruthenica than in $M$. truncatula under drought stress (Fig. 6, Additional file 1: Figure S20B). The large-scale expansion of FHY3/FAR1 family may confer $M$. ruthenica great tolerant to abiotic stress among the legume forages examined in our study. In addition, our results also reveal that AP2/ERF family, including DREB TFs, may contribute to the most droughtresponsive members in $M$. ruthenica and $M$. truncatula (Fig. 3c, Additional file 1: Figure S20C).

Traits associated with high yield have been paid great attention during the process of domestication, thus rendering the cultivated alfalfa less tolerant to environmental stress due to the loss of traits involved in adaptation to harsh environments [32]. We identified the lost genes in cultivated $M$. truncatula and $M$. sativa during domestication (Additional file 2: Table S22, Additional file 2: Table S23). More genes were lost in M. truncatula compared with M. sativa. Most enriched GO terms based on lost genes in $M$. sativa during domestication were also enriched in M. sativa. In the GO term of "nucleic acid binding," FHY3/FAR1 genes were identified, which is consistent with the transcription factor analysis among the three species (Fig. 3a). In addition, we found that DNA helicase genes represented high proportion in the GO term "DNA repair." This gene family codes for molecular motor proteins in various cellular mechanisms and regulators in the pre-mRNA splicing and plays important roles in alleviating multiple abiotic stresses [33]. The Helicase 45 of pea is responsive to salinity, dehydration, and low temperature. Expression of this gene confers transgenic plants more tolerance to abiotic stress [34-36]. It is expected that the lost genes associated with stress response, transcriptional regulation, and DNA repair may explain the less tolerance of two cultivated species than wild $M$. ruthenica.

The great variations in drought tolerance among the $M$. ruthenica accessions allowed us to explore mechanisms underlying the tolerance of $M$. ruthenica to drought. The identified candidate genes associated with drought tolerance were involved in photosynthesis and stress response (Additional file 2: Table S29). The photosynthetic electron transport chain is suppressed under drought stress, thus leading to massive accumulation of ROS. Accessions of $M$. ruthenica with a greater drought tolerance may be equipped with more efficient photosynthesis systems, thus allowing $M$. ruthenica to supply energy by maintaining relatively high photosynthetic rates under drought stress (Fig. 6).

We further explored the mechanisms underlying the greater tolerance of Zhenglanqi accession than that of Xinghe accession by a transcriptomic approach. The two closely correlative GO terms with drought tolerance (GO: 0006950, response to stress; GO: 0006560, proline metabolic process) were enriched in the Zhenglanqi accession, but not in the Xinghe accession (Fig. 5, Additional file 2: Table S30, Additional file 2: S31). Moreover, the common GO terms of nucleic acid-binding transcription factor activity in Zhenglanqi accession showed higher degree of enrichment. To identify the potential target genes of TFs in the two accessions, we analyzed the promoter elements of all the responsive-genes. We identified the involvements of the target genes of MYB TFs in detoxification (e.g., $P O D$ ) and proline synthesis (e.g., P5CS). The less accumulation of $\mathrm{H}_{2} \mathrm{O}_{2}$ and greater accumulation of proline in the Zhenglanqi accession may be accounted for by the GO enrichment of peroxidase activity and proline biosynthetic process (Fig. 6, Additional file 1: Figure S20D, Additional file 1: Figure S20E, Additional file 2: Table S32). Moreover, the observation that more target genes of MYB in the GO terms of the Zhenglanqi accession may suggest important roles of MYB transcription factors in drought tolerance of $M$. ruthenica (Additional file 2: Table S32).

Medicago ruthenica has capacious prospects to provide resource for improving traits associated with tolerance to environmental stress in alfalfa. The alfalfa genome 


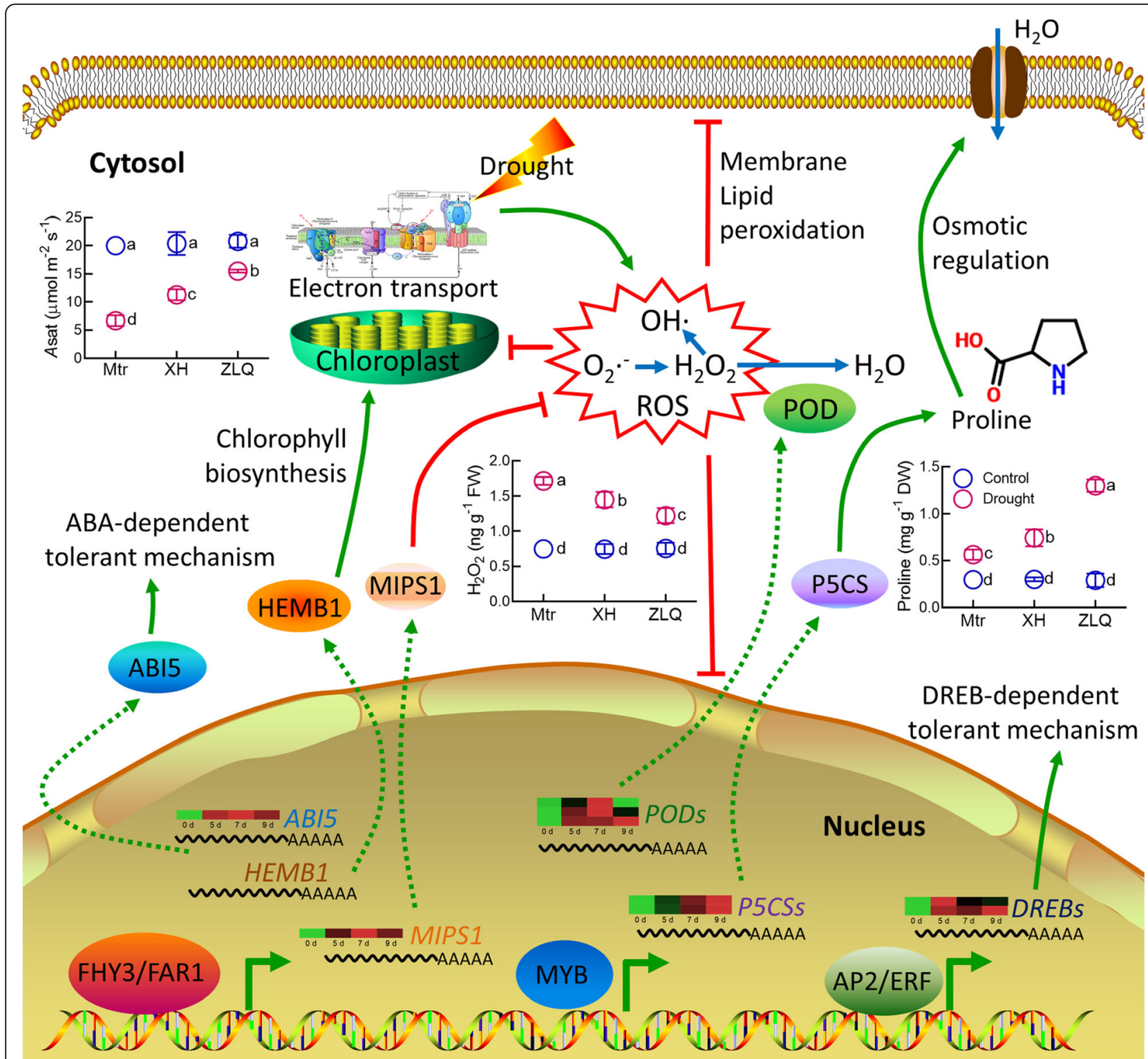

Fig. 6 Proposed mechanisms behind tolerance of M. ruthenica to drought stress. The dotted lines represent the transfer and translation. Arrows denote positive effects, whereas lines ending with a short bar indicate negative effects. Photosynthetic rates were measured using the newly expanded leaves treated for 7 days by a gas exchange system (LI-COR 6800). Root samples at the seventh day of drought were used to determine $\mathrm{H}_{2} \mathrm{O}_{2}$ and proline. Asat photosynthetic rate, ROS reactive oxygen species, Mtr M. truncatula, XH Xinghe accession, and ZLQ Zhenglangi accession. Data are means \pm SE $(n=4)$. Different letters mean significant differences among treatments at $P<0.05$

will greatly push this development $[14,15]$. The assembly of $M$. ruthenica genome opens a new avenue for genomic- and genetic-based improvement of legume forage, particularly for elucidation of molecular mechanisms associated with response and adaptation to environmental stress for alfalfa. In the present study, we also identified lost genes in cultivated alfalfa and $M$. truncatula during domestication, and stress-selective/responsive genes in $M$. ruthenica, which can be used in the tolerant trait improvement of cultivated alfalfa.
Induced distant hybridization of alfalfa and M. ruthenica may be an effective way to breed new cultivated species. Innovative breeding technology can be used in breeding alfalfa with great tolerance to harsh environments. Recent advances in CRISPR/Cas genome editing enable effectively targeted modification in most crops, thus accelerating crop improvement [37]. The specifically expressional genes in $M$. ruthenica under stress and the lost genes of alfalfa during domestication can be candidate genes for transforming alfalfa using CRISPR/Cas9 
system for breeding tolerant varieties. The genome information and identification of resistance genes of $M$. ruthenica can shed important light on the mechanisms behind the tolerance of $M$. ruthenica to environmental stress and provide valuable resource for improvements of agronomic traits associated with high yield and great tolerance to environmental stress through a molecular breeding approach.

\section{Conclusions}

In this study, we assembled a high-quality reference genome for a perennial legume forage of $M$. ruthenica and explored the molecular mechanisms by which $M$. ruthenica adapts to the drought stress using genomic and genetic approaches. Specifically, we analyzed transcription factors, expanded/contracted gene families, and retained genes compared with cultivated Medicago species, SNP variations, and drought-responsive genes by comparative genomics, resequencing, and transcriptomics. Therefore, the genome of $M$. ruthenica provides new insights into the tolerant mechanisms of legume forage to environmental stress and valuable information for genetic-based improvements of agronomic traits in perennial legume crops.

\section{Methods}

\section{Sequencing and assembly}

Leaf samples from a single plant of $M$. ruthenica collected from Xinghe County $\left(40^{\circ} 56^{\prime} 2^{\prime \prime} \mathrm{N}, 113^{\circ} 31^{\prime} 4^{\prime \prime}\right.$ E) in northern China were used for the genome sequencing. This accession of $M$. ruthenica is of a typical phenotype and distributed widely in China. A total of $76.54 \mathrm{~Gb}$ of reads from $270 \mathrm{bp}$ and $500 \mathrm{bp}$ insert size libraries were used to calculate the k-mer frequency distribution. The 17-mer sequences of sliding windows were used to estimate $M$. ruthenica genome size (the total number of $\mathrm{k}$-mer/the depth of the major peak). The heterozygosity ratio of the $M$. ruthenica genome was estimated using GenomeScope based on the 17-mer data (http://qb.cshl.edu/genomescope/). De novo assembly of PacBio SMRT reads was performed using FALCON [38]. Thereafter, Pilon was used to perform the second round of error correction with the Illumina paired-end reads [39]. The Purge Haplotigs (https:// bitbucket.org/mroachawri/purge_haplotigs) were used to remove the redundant sequences caused by heterozygosity. The scaffolding was performed by $10 \times$ Gscaff v2.1 using $10 \times$ Genomics data [40]. SSPACE v3.0 was used to build scaffolds using Illumina data from all the matepair libraries [41]. The assembly was used as input for scaffolding by fragScaff along with an N-base bed file and a repeat bed file produced by self-against-self BlastN [42]. The Hi-C data were mapped to the original scaffold genome using BWA v0.7.7 [43], and only reads with unique alignment positions were extracted to construct a chromosome scale assembly using LACHESIS [44]. The completeness of the assembly was assessed using BUSCO and CEGMA $[45,46]$.

\section{Annotation}

Repetitive sequences include transposable elements (TEs) and tandem repeats. RepeatMasker v3.3.0 [47] was performed to detect TEs by comparing sequences with integrate repeat libraries including known repeat library (Repbase 15.02) and the de novo repeat library built by RepeatModeler v1.0.5, RepeatScout3, and LTR-Finder4. Tandem repeats were ascertained in the genome using Tandem Repeats Finder [48]. De novo predictions, homolog-based, and RNA-seq-based predictions were employed to annotate the protein-coding genes. Five ab initio gene prediction programs were used to predict genes, including Augustus v3.0.2, Genescan v1.0, Geneid, GlimmerHMM v3.0.2, and SNAP. Protein sequences of ten homologous species $A$. thaliana, M. truncatula, G. max, $T$. pratense, $C$. arietinum, Vigna angularis, $V$. radiate, C. cajan, P. vulgaris, and Arachis ipaensis were downloaded from the Ensembl or NCBI. Homologous sequences were aligned against to the repeat-masked $M$. ruthenica genome using TblastN ( $e$ value $\leq 1 \mathrm{e}^{-5}$ ). Genewise v2.2.0 was employed to predict gene models based on the alignment sequences [49]. There were two ways to assemble the RNA-seq data into the unique sequences of transcripts. One was mapping the RNA-seq data to the $M$. ruthenica genome using Tophat v2.0.8 [50] and Cufflinks v2.1.1 [51] for transcript assembly. The other was applying Trinity [52] to assemble the RNA-seq data, and then PASA software [53] improved the gene structures. A weighted and non-redundant gene set was generated by EVidenceModeler [54] which merged all genes models predicted by the above three approaches. PASA adjusted the gene models generated by EVM. Lastly, the gene sets were filtered according to the following standards: coding region lengths of amino acids $\leq 50$, supported only by de novo methods and with $\mathrm{FPKM}<5$. Functional annotation of protein-coding genes was obtained according to best Blast hit by BlastP $\left(e\right.$ value $\left.\leq 1 \mathrm{e}^{-5}\right)$ against SwissProt [55] and NCBI non-redundant (NR) protein databases. Motifs and domains were annotated by using InterProScan v4.7 [56] to search against InterPro v29.0 databases [56], including Pfam, Prints, Prosite, ProDom, and Smart. The tRNA genes were predicted by tRNAscan-SE software [57]. The miRNA and snRNA fragments were identified by INFERNAL software [58] against the Rfam database [59]. The rRNAs were found by using BlastN ( $e$ value $\leq 1 \mathrm{e}^{-10}$ ) against invertebrate rRNA database. The structure figure was drawn along eight chromosomes of $M$. ruthenica genome using Circos program [60]. To estimate the assembly of genome, transcriptome data 
from roots, stems, leaves, flowers, and pods were mapped to the genome assembly using Hisat2 [61], and transcripts were assembled using Trinity [52]. The unigene, which was the longest transcript selected from Trinity, was aligned to the genome assembly by Blat [62].

\section{Genome comparison and evolution}

Gene families were generated by OrthoMCL [63]. Nucleotide and protein data of the other six species were downloaded from the Ensembl or NCBI. We selected the first group of allelic chromosomes (chr1.1-chr1.8) of M. sativa for analysis [14]. Before an "all against all" BlastP ( $e$ value $\leq 1 \mathrm{e}^{-7}$ ) program, the longest transcript was selected from alternative splicing transcripts belonging to one gene. The alignments with high-scoring segment pairs were conjoined for each gene pair by Solar [64]. To identify homologous gene-pairs, more than $30 \%$ coverage of the aligned regions was required. Finally, the alignments were clustered into gene families using OrthoMCL with 1.5 inflation index. GO enrichment of unique gene families in $M$. ruthenica were analysed. The shared single-copy orthologs were utilized to construct the phylogenetic tree. Protein sequences of these orthologs were aligned by muscle [65], then protein alignments were transformed to coding sequence (CDS) alignments. The phylogenetic tree was constructed by the ML (maximum likelihood) TREE algorithm in RAxML v7.2.3 [66]. The mcmctree program of Paml [67] was applied to estimate divergence time among 7 species. One calibration point was selected as normal priors to restrain the age of the nodes (89.8-125 Mya for A. thaliana-G. max). Mcscan [68] was used to construct the genome synteny within $M$. ruthenica, and among species of $M$. truncatula, $C$. arietinum, $T$. pretense, and $M$. sativa, respectively. We selected the first group of allelic chromosomes (chr1.1-chr1.8) of $M$. sativa for this analysis [14]. Syntenic blocks containing at least 5 genes were obtained based on the similarity gene pairs (Blastp: $e<1 \mathrm{e}^{-5}$ ). We extracted all the duplicated gene pairs from syntenic blocks and calculated the 4DTv (transversion substitutions at fourfold degenerate sites) distance. The genome synteny between $M$. ruthenica and cultivated alfalfa at the diploid level (https:// legumeinfo.org/data/public/Medicago_sativa/CADL_ HM342.gnm1.rVNY/) was constructed using Nucmer (http://mummer.sourceforge.net/). CAFE was used to analyze gene family expansion [69]. Transcription factors of $M$. ruthenica, $M$. truncatula, and $M$. sativa were identified using iTAK software [70]. Phylogenetic tree of FHY3/FAR1 family was drawn by FastTree software.

The genome of $M$. ruthenica was used to analyze the genome variation of $M$. truncatula and $M$. sativa during domestication. The gene pairs between reference and target species were obtained according to best hit by
BlastP ( $e$ value $\leq 1 \mathrm{e}^{-5}$ ). These gene pairs were sorted by linear order along chromosomes in the genome of target species. If there was one gene in the genome of target species, but no corresponding gene in the reference genome, the absent gene in the reference genome was marked "NA". The absent region was defined as the upstream and downstream of "NA". We used Exonerate software [71] to predict homolog-based gene on this region. If the genes of the target species were not predicted in the genome of reference species, it indicated that the genes of target species were specifically retained genes, and these genes were lost in the reference species.

\section{Survival rate and experiments of physiology}

Seedlings of $T$. pretense, $M$. ruthenica, $M$. truncatula, $M$. sativa, $M$. varia, and $M$. falcata were grown in pots $(7 \times 7 \times 12 \mathrm{~cm})$ filled with vermiculite and peat soil $(2: 1)$ under controlled conditions $\left(26^{\circ} \mathrm{C}\right.$ day $/ 20^{\circ} \mathrm{C}$ night, $16 \mathrm{~h}$ photoperiod). Drought stress was initiated by withholding water supply to 6 -week-old seedlings for varying periods after seedlings were fully watered. In the comparison of $M$. ruthenica with other legume species, the phenotypes were photographed at the tenth day of drought. The survival rate at the tenth day of drought treatment was determined after re-watering for 7 days. In the comparison of $20 \mathrm{M}$. ruthenica accessions from different locations, the phenotypes were photographed at the twelfth day of drought, and survival rate at the twelfth day of drought was counted using the identical protocols. Four biological replications and 15 seedlings in each biological replication were used to determine survival rate. Photosynthetic rates were measured using the newly expanded leaves treated for 7 days by a gas exchange system (LI-COR 6800). Air flow rate and $\mathrm{CO}_{2}$ concentration were maintained at $750 \mu \mathrm{mol} \mathrm{s}^{-1}$ and 400 $\mu \mathrm{mol} \mathrm{mol}{ }^{-1}$, respectively. Root samples at the seventh day of drought were used to determine $\mathrm{H}_{2} \mathrm{O}_{2}$ and proline $[72,73]$.

\section{Analysis of SNPs}

Leaf samples of 19 accession of M. ruthenica were used for resequencing. Genomic DNA was isolated from these materials. Randomly fractured DNA was used to construct libraries using TruSeq Library Construction Kit (Illumina), and raw data were obtained using Illumina Hiseq PE150. The adapter sequences of the raw reads were trimmed, and low-quality reads were filtered to obtain clean data. Clean data were mapped to the genome of $M$. ruthenica using BWA [43]. The high-quality SNPs were screened using SAMTools [74]. SNPs were annotated using ANNOVAR [75]. Phylogenetic tree was constructed by neighbor-joining method with bootstrap values from a minimum of 1000 trials using TreeBeST software. According to the survival rate under drought stress, the top 7 
tolerant accessions (Zhenglanqi, Chifeng, Yulin, Jiuquan, Shangdu, Manzhouli, and Zhengxiangbaiqi) were grouped to tolerance population, and the least 7 tolerant accessions were (Chicheng, Baode, Fanshi, Qiqihar, Honggang, Gulang, and Shenchi) grouped to control population. The population differentiation (Fst) [76] and nucleotide diversity $(\theta \pi)$ [77] were calculated using SNP data across $40 \mathrm{~kb}$ windows with a 20-kb slide. The SNP windows were screened using top $5 \%$ Fst and $\log _{2}\left(\theta \pi_{\text {Control }} / \theta \pi_{\text {Tolerance }}\right)$. The genes in these windows were identified to perform GO enrichment [78].

\section{Identification and analysis of drought-responsive genes} Root samples of $M$. ruthenica accessions from the sites of Zhenglanqi and Xinghe at the 5th, 7th, and 9th day under drought were harvested to construct transcriptome libraries. Three biological replicates were used for each treatment. The mRNA enriched from total RNA was used to construct RNA-seq libraries using TruSeq RNA Sample Preparation Kits. The libraries were sequenced on an Illumina Hiseq PE150 platform and paired-end reads $(2 \times 150 \mathrm{bp})$ were generated. The adapter sequences of the raw reads were trimmed, and low-quality reads were filtered to obtain clean data. Differential expression analysis was performed using the DESeq $\mathrm{R}$ package [79]. The resulting $P$ values were adjusted using the Benjamini and Hochberg's approach for controlling the false discovery rate. Genes with an adjusted $P$ value $<0.05$ by DESeq were assigned as differentially expressed. From the data of Xinghe accessions at the 7th day under drought stress, drought-responsive TF genes were identified and drought-responsive TF genes of $M$. truncatula under the same treatment were identified using the published data [80]. STRING v11 [81] were used to build protein association networks, and figures were drawn by Cytoscape [82]. The GO terms of drought-responsive genes were analyzed in two accessions. Rich factor is the proportion of the differentially expressed gene number to the total gene number in a certain GO term. The protein sequence alignment was performed by RAxML with homologous proteins of other species [66]. The cis-acting regulatory elements were searched by PLACE [83]. The drought-responsive potential MYB-target-genes with more than two MYBcore elements were analyzed for both accessions using GO.

\section{Supplementary Information}

The online version contains supplementary material available at https://doi. org/10.1186/s12915-021-01033-0.

Additional file 1: Figure S1. Distribution of 17-mer frequency and heterozygosity estimate of $\mathbf{M}$. ruthenica genome. Figure $\mathbf{S 2}$. Hi-C interactome within and among chromosomes (Mru1-Mru8). Figure $\mathbf{S 3 .}$
Distribution of GC content and depth. Figure S4. Divergence distribution of classified families of transposable elements. Figure S5. Comparison of gene parameters among Medicago ruthenica genome and other genomes. Figure S6. The distribution of genes in different species. Figure S7. The density comparison of transposable elements between $M$. ruthenica and $M$. truncatula. Figure S8. Genome synteny between $M$. ruthenica and $M$. truncatula. Figure S9. Synteny analysis between genomes of M. ruthenica and C. arietinum. Figure S10. Synteny analysis between genomes of $M$. ruthenica and T. pretense. Figure S11. Synteny between $M$. ruthenica and $M$. sativa in the level of scaffolds. Figure S12. Distribution of FHY3/FAR1 genes and synteny analysis within M. ruthenica genome.

Figure S13. The survival rate comparison of other legume forages and M. ruthenica under drought stress. Figure S14. Principal component analysis plot of genetic variation. Figure $\mathbf{S 1 5}$. The genetic structure of populations. Figure S16. Selective sweep analysis using Fst \& Өп. Figure S17. Venn diagram of drought-responsive genes from two M. ruthenica accessions collected from sites of Zhenglanqi and Xinghe. Figure S18. The predicted interaction of proteins encoded by drought-responsive genes in $\mathbf{M}$. ruthenica. Figure S19. The number of genes present in the duplicated paralogous blocks of $M$. ruthenica and M. sativa. Figure S20. The expression of MrABI5, MrMIPS1, MrDREBs, MrPODs and MrP5CSs in two accessions under drought stress.

Additional file 2: Table S1. Estimation of genome size of $M$. ruthenica using 17-mer analysis. Table S2. Sequencing data generated from different platforms/strategies. Table $\mathbf{S 3}$ The assemblage summary of the $M$. ruthenica genome Version 0.8 before chromosome anchoring by $\mathrm{Hi}-\mathrm{C}$. Table S4. The assemblage summary of the $M$. ruthenica genome Version 1.0. Table S5. Genome estimation using RNA sequencing data. Table S6. Assessment of the transcript coverage with the transcritome assembly. Table S7. The prediction of repeat elements in the M. ruthenica genome. Table S8. Categories of transposable elements predicted in the $M$. ruthenica genome. Table S9. General statistics of predicted proteincoding genes in M. ruthenica. Table S10. General statistics of mapping rate to functional database of protein-coding genes. Table S11. General statistics of protein-coding genes of homolog species. Table S12. GO enrichment of unique genes in M. ruthenica. Table S13. General statistics of non-coding RNA of the genome. Table S14. Statistics of synteny blocks within M. ruthenica and M. truncatula, M. ruthenica and T. pratense, $M$. ruthenica and C. arietinum, and $M$. ruthenica itself. Table S15. The corresponding relationship of synteny blocks in $M$. ruthenica with $M$. truncatula. Table S16. Statistics of TFs in M. ruthenica, M. truncatula and $M$. sativa. Table S17. Information of FHY3/FAR1 genes in M. ruthenica. Table S18. Information of FHY3/FAR1 genes in M. truncatula. Table S19. The GO enrichments of expanded families. Table S20. The GO enrichments of contracted families. Table S21. The GO enrichments of lost genes in the genome of $M$. truncatula during domestication. Table S22. The GO enrichments of lost genes in the genome of $M$. sativa during domestication. Table S23. Statistics of transcriptome libraries. Table S24. TFs responsive to drought in M. ruthenica. Table S25. TFs responsive to drought in M. truncatula. Table S26. Resequencing data of 19 accessions. Table S27. Statistics and annotation of SNPs. Table S28. Candidate genes associated with drought tolerance analyzed by selective sweep. Table S29. The GO enrichments of candidate genes associated with drought tolerance analyzed by selective sweep. Table S30. The GO enrichments of drought-responsive genes from Zhenglangi accession. Table S31. The GO enrichments of drought-responsive genes from Xinghe accession. Table S32. Classification of potential MYB target genes which were responsive to drought stress.

\section{Acknowledgements}

We thank Dr. Bao Nie (Institute of Botany, the Chinese Academy of Sciences) who provides Shenchi accession of M. ruthenica. We thank Dr. Yuannian Jiao (Institute of Botany, the Chinese Academy of Sciences) for his help in the analysis of genome evolution. We thank the anonymous reviewers and editors for their constructive suggestions on our previous versions of the manuscript.

\section{Authors' contributions}

TW and WHZ conceived the study and jointly wrote the manuscript. TW and LR collected materials. TW, LR, CL, DZ, and XZ performed experiments of 
plant physiology. MZ, GZ, and DG generated genome and transcriptome of $M$. ruthenica. TW and MZ performed bioinformatic analyses. ADF and NDY supplied the genome data of cultivated alfalfa at the diploid level. RC, YC, ZW, FS, and YL edited the manuscript. The authors analyzed the data and approved the final manuscript.

\section{Funding}

The research was supported by the National Natural Science Foundation of China $(32070351,31830011)$ and Chinese Academy of Sciences (KFJ-STSZDTP-056).

\section{Availability of data and materials}

The assembled genome, transcriptome, and resequencing data have been deposited in GenBank under accession WNNG00000000 [84], PRJNA589938 [85] and PRJNA598783 [86], respectively. The files of genome assembly and annotation have also been deposited in figshare (https://doi.org/10.6084/m9. figshare.12726932) [87].

\section{Declarations}

\section{Ethics approval and consent to participate}

Not applicable.

\section{Consent for publication}

Not applicable.

\section{Competing interests}

The authors declare no competing interests.

\section{Author details}

'State Key Laboratory of Vegetation and Environmental Change, Institute of Botany, The Chinese Academy of Sciences, Beijing, China. ${ }^{2}$ Novogene Bioinformatics Institute, Beijing, China. ${ }^{3}$ School of Life Sciences, Lanzhou University, Lanzhou, China. ${ }^{4}$ Institute of Grassland Research, Chinese Academy of Agricultural Sciences, Huhehot, China. ${ }^{5}$ College of Ecology and Environmental Science, Inner Mongolia Agricultural University, Huhehot China. ${ }^{6}$ National Centre for Genome Resources, Santa Fe, New Mexico, USA. ${ }^{7}$ Institute of Vegetables and Flowers, Chinese Academy of Agricultural Sciences, Beijing, China. ${ }^{8}$ Departments of Plant Pathology and Plant Biology, University of Minnesota, Minnesota, USA. ${ }^{9}$ Inner Mongolia Research Centre for Prataculture, The Chinese Academy of Sciences, Beijing, China.

Received: 2 September 2020 Accepted: 21 April 2021

Published online: 06 May 2021

\section{References}

1. Small E, Jomphe M. A synopsis of the genus Medicago (Leguminosae). Can J Bot. 1989;67(11):3260-94. https://doi.org/10.1139/b89-405.

2. Li HY, Li ZY, Cai LY, Shi WG, Mi FG, Shi FL. Analysis of genetic diversity of Ruthenia Medic (Medicago ruthenica (L.) Trautv.) in Inner Mongolia using ISSR and SSR markers. Genet Resour Crop Ev. 2013;60(5):1687-94. https://doi. org/10.1007/s10722-012-9950-3.

3. Campbell TA, Bao G, Xia ZL. Agronomic evaluation of Medicago ruthenica collected in Inner Mongolia. Crop Sci. 1997;37(2):599-604. https://doi.org/1 0.2135/cropsci1997.0011183X003700020048x.

4. Balabaev GA. Yellow lucernes of Siberia, Medicago ruthenica (L.) Ledb. and M. platycarpa (L.) Ledb. Bull App Bot Genet Plant Breed Serv. 1934;7:13-123.

5. Benedito VA, Torres-Jerez I, Murray JD, Andriankaja A, Allen S, Kakar K, et al. A gene expression atlas of the model legume Medicago truncatula. Plant J. 2008:55(3):504-13. https://doi.org/10.1111/j.1365-313X.2008.03519.x.

6. Bouton JH. Breeding lucerne for persistence. Crop Pasture Sci. 2012;63(2): 95-106. https://doi.org/10.1071/CP12009.

7. Collino DJ, Dardanelli JL, De Luca MJ, Racca RW. Temperature and water availability effects on radiation and water use efficiencies in alfalfa (Medicago sativa L.). Aust J Exp Agr. 2005;45(4):383-90. https://doi.org/10.1071/EA04050.

8. Cornacchione MV, Suarez DL. Emergence, forage production, and ion relations of alfalfa in response to saline waters. Crop Sci. 2015;55(1):444-57. https://doi.org/10.2135/cropsci2014.01.0062.

9. Zhang W, Hou L, Yang J, Song S, Mao X, Zhang Q, et al. Establishment and management of alfalfa pasture in cold regions of China. Chin Sci Bull. 2018; 63(17):1651-63. https://doi.org/10.1360/N972017-01181.
10. Dear BS, Reed KFM, Craig AD. Outcomes of the search for new perennial and salt tolerant pasture plants for southern Australia. Aust J Exp Agr. 2008; 48(4):578-88. https://doi.org/10.1071/EA07118.

11. Sato S, Nakamura Y, Kaneko T, Asamizu E, Kato T, Nakao M, et al. Genome structure of the legume, Lotus japonicus. DNA Res. 2008;15(4):227-39. https://doi.org/10.1093/dnares/dsn008.

12. Young ND, Debelle F, Oldroyd GED, Geurts R, Cannon SB, Udvardi MK, et al. The Medicago genome provides insight into the evolution of rhizobial symbioses. Nature. 2011;480(7378):520-4. https://doi.org/10.1038/nature10625.

13. De Vega JJ, Ayling S, Hegarty M, Kudrna D, Goicoechea JL, Ergon A, et al. Red clover (Trifolium pratense L.) draft genome provides a platform for trait improvement. Sci Rep-Uk. 2015;5(1):17394. https://doi.org/10.1038/ srep17394.

14. Chen $\mathrm{H}$, Zeng $Y$, Yang $Y$, Huang L, Tang B, Zhang H, et al. Allele-aware chromosome-level genome assembly and efficient transgene-free genome editing for the autotetraploid cultivated alfalfa. Nat Commun. 2020;11(1): 2494. https://doi.org/10.1038/s41467-020-16338-x.

15. Shen C, Du H, Chen Z, Lu H, Zhu F, Chen H, et al. The chromosome-level genome sequence of the autotetraploid alfalfa and resequencing of core germplasms provide genomic resources for alfalfa research. Mol Plant. 2020; 13(9):1250-61. https://doi.org/10.1016/j.molp.2020.07.003.

16. Liu M, Wang TZ, Zhang WH. Sodium extrusion associated with enhanced expression of SOS1 underlies different salt tolerance between Medicago falcata and Medicago truncatula seedlings. Environ Exp Bot. 2015;110:46-55. https://doi.org/10.1016/j.envexpbot.2014.09.005.

17. Yu LQ. Systematical evaluation on alfalfa germplasm and genetic diversity analysis. Huhehot: Inner Mongolia Agricultural University; 2009.

18. Wang DK, Li H, Luo XY. Crossbreeding of Melilotoides ruthenicus and Medicago sativa. Acta Agrestia Sinica. 2008;16:458-65.

19. Schmutz J, McClean PE, Mamidi S, Wu GA, Cannon SB, Grimwood J, et al. A reference genome for common bean and genome-wide analysis of dual domestications. Nat Genet. 2014;46(7):707-13. https://doi.org/10.1038/ng.3008.

20. Schmutz J, Cannon SB, Schlueter J, Ma JX, Mitros T, Nelson W, et al. Genome sequence of the palaeopolyploid soybean. Nature. 2010;463(7278): 178-83. https://doi.org/10.1038/nature08670.

21. Varshney RK, Chen WB, Li YP, Bharti AK, Saxena RK, Schlueter JA, et al. Draft genome sequence of pigeonpea (Cajanus cajan), an orphan legume crop of resource-poor farmers. Nat Biotechnol. 2012;30(1):83-9. https://doi.org/10.1 038/nbt.2022.

22. Varshney RK, Song C, Saxena RK, Azam S, Yu S, Sharpe AG, et al. Draft genome sequence of chickpea (Cicer arietinum) provides a resource for trait improvement. Nat Biotechnol. 2013;31(3):240-6. https://doi.org/10.1038/nbt.2491.

23. Wu Z, Hou X, Ren W, Du J, Zhao Q, Wang Z. Prediction of the potential distribution of Medicago ruthenica in China under climate change. Acta Agrestia Sinica. 2018;26:898-906.

24. Yoder JB, Briskine R, Mudge J, Farmer A, Paape T, Steele K, et al. Phylogenetic signal variation in the genomes of Medicago (Fabaceae). Syst Biol. 2013;62(3):424-38. https://doi.org/10.1093/sysbio/syt009.

25. Albert VA, Barbazuk WB, dePamphilis CW, Der JP L-MJ, Ma H, Palmer JD, et al. The Amborella genome and the evolution of flowering plants. Science. 2013:342:1241089.

26. Wu SD, Han BC, Jiao YN. Genetic contribution of paleopolyploidy to adaptive evolution in angiosperms. Mol Plant. 2020;13(1):59-71. https://doi. org/10.1016/j.molp.2019.10.012.

27. Cannon SB, McKain MR, Harkess A, Nelson MN, Dash S, Deyholos MK, et al. Multiple polyploidy events in the early radiation of nodulating and nonnodulating legumes. Mol Biol Evol. 2015;32(1):193-210. https://doi.org/1 0.1093/molbev/msu296.

28. Rispe C, Legeai F, Nabity PD, Fernandez R, Arora AK, Baa-Puyoulet P, et al. The genome sequence of the grape phylloxera provides insights into the evolution, adaptation, and invasion routes of an iconic pest. BMC Biol. 2020; 18(1):90. https://doi.org/10.1186/s12915-020-00820-5.

29. Wang $H$, Wang HY. Multifaceted roles of FHY3 and FAR1 in light signaling and beyond. Trends Plant Sci. 2015;20(7):453-61. https://doi.org/10.1016/j. tplants.2015.04.003.

30. Tang WJ, Ji Q, Huang YP, Jiang ZM, Bao MZ, Wang HY, et al. FAR-RED ELONGATED HYPOCOTYL3 and FAR-RED IMPAIRED RESPONSE1 transcription factors integrate light and abscisic acid signaling in Arabidopsis. Plant Physiol. 2013;163(2):857-66. https://doi.org/10.1104/pp.113.224386.

31. Ma L, Tian T, Lin RC, Deng XW, Wang HY, Li G. Arabidopsis FHY3 and FAR1 regulate light-induced myo-inositol biosynthesis and oxidative stress 
responses by transcriptional activation of MIPS1. Mol Plant. 2016;9(4):541-57. https://doi.org/10.1016/j.molp.2015.12.013.

32. Brozynska M, Furtado A, Henry RJ. Genomics of crop wild relatives: expanding the gene pool for crop improvement. Plant Biotechnol J. 2016; 14(4):1070-85. https://doi.org/10.1111/pbi.12454.

33. Pascuan C, Frare R, Alleva K, Ayub ND, Soto G. mRNA biogenesis-related helicase elF4AIll from Arabidopsis thaliana is an important factor for abiotic stress adaptation. Plant Cell Rep. 2016;35(5):1205-8. https://doi.org/10.1007/ s00299-016-1947-5.

34. Amin M, Elias SM, Hossain A, Ferdousi A, Rahman MS, Tuteja N, et al. Overexpression of a DEAD-box helicase, PDH45, confers both seedling and reproductive stage salinity tolerance to rice (Oryza sativa L.). Mol Breeding. 2012;30(1):345-54. https://doi.org/10.1007/s11032-011-9625-3.

35. Augustine SM, Narayan JA, Syamaladevi DP, Appunu C, Chakravarthi M, Ravichandran $V$, et al. Introduction of pea DNA Helicase 45 into Sugarcane (Saccharum spp. Hybrid) enhances cell membrane thermostability and upregulation of stress-responsive genes leads to abiotic stress tolerance. Mol Biotechnol. 2015;57(5):475-88. https://doi.org/10.1007/s12033-015-9841-x.

36. Shivakumara TN, Sreevathsa R, Dash PK, Sheshshayee MS, Papolu PK, Rao U, et al. Overexpression of pea DNA Helicase 45 (PDH45) imparts tolerance to multiple abiotic stresses in chili (Capsicum annuum L.). Sci Re. 2017;7:2760.

37. Chen KL, Wang YP, Zhang R, Zhang HW, Gao CX. CRISPR/Cas genome editing and precision plant breeding in agriculture. Annu Rev Plant Biol. 2019;70(1):667-97. https://doi.org/10.1146/annurev-arplant-050718-100049.

38. Chin CS, Peluso P, Sedlazeck FJ, Nattestad M, Concepcion GT, Clum A, et al. Phased diploid genome assembly with single-molecule real-time sequencing. Nat Methods. 2016;13(12):1050-4. https://doi.org/10.1038/nmeth.4035.

39. Walker BJ, Abeel T, Shea T, Priest M, Abouelliel A, Sakthikumar S, et al. Pilon: an integrated tool for comprehensive microbial variant detection and genome assembly improvement. PLoS One. 2014;9(11):e112963. https://doi. org/10.1371/journal.pone.0112963.

40. Mostovoy Y, Levy-Sakin M, Lam J, Lam ET, Hastie AR, Marks P, et al. A hybrid approach for de novo human genome sequence assembly and phasing. Nat Methods. 2016;13(7):587-90. https://doi.org/10.1038/nmeth.3865.

41. Boetzer M, Henkel CV, Jansen HJ, Butler D, Pirovano W. Scaffolding preassembled contigs using SSPACE. Bioinformatics. 2011;27(4):578-9. https:// doi.org/10.1093/bioinformatics/btq683.

42. Adey A, Kitzman JO, Burton JN, Daza R, Kumar A, Christiansen L, et al. In vitro, long-range sequence information for de novo genome assembly via transposase contiguity. Genome Res. 2014;24(12):2041-9. https://doi.org/1 $0.1101 /$ gr.178319.114.

43. Li H, Durbin R. Fast and accurate short read alignment with BurrowsWheeler transform. Bioinformatics. 2009;25(14):1754-60. https://doi.org/10.1 093/bioinformatics/btp324.

44. Burton JN, Adey A, Patwardhan RP, Qiu RL, Kitzman JO, Shendure J. Chromosome-scale scaffolding of de novo genome assemblies based on chromatin interactions. Nat Biotechnol. 2013;31(12):1119-25. https://doi. org/10.1038/nbt.2727.

45. Simao FA, Waterhouse RM, loannidis P, Kriventseva EV, Zdobnov EM BUSCO: assessing genome assembly and annotation completeness with single-copy orthologs. Bioinformatics. 2015;31(19):3210-2. https://doi.org/1 0.1093/bioinformatics/btv351

46. Parra G, Bradnam K, Korf I. CEGMA. a pipeline to accurately annotate core genes in eukaryotic genornes. Bioinformatics. 2007;23(9):1061-7. https://doi. org/10.1093/bioinformatics/btm071.

47. Chen N. Using RepeatMasker to identify repetitive elements in genomic sequences. Curr Protoc Bioinformatics. 2004;4:Unit4.10.

48. Benson G. Tandem repeats finder: a program to analyze DNA sequences. Nucleic Acids Res. 1999;27(2):573-80. https://doi.org/10.1093/nar/27.2.573.

49. Birney E, Clamp M, Durbin R. GeneWise and genomewise. Genome Res. 2004;14(5):988-95. https://doi.org/10.1101/gr.1865504

50. Trapnell C, Pachter L, Salzberg SL. TopHat: discovering splice junctions with RNA-Seq. Bioinformatics. 2009;25(9):1105-11. https://doi.org/10.1093/ bioinformatics/btp120.

51. Trapnell C, Williams BA, Pertea G, Mortazavi A, Kwan G, van Baren MJ, et al. Transcript assembly and quantification by RNA-Seq reveals unannotated transcripts and isoform switching during cell differentiation. Nat Biotechnol. 2010;28(5):511-5. https://doi.org/10.1038/nbt.1621.

52. Grabherr MG, Haas BJ, Yassour M, Levin JZ, Thompson DA, Amit I, et al. Fulllength transcriptome assembly from RNA-Seq data without a reference genome. Nat Biotechnol. 2011;29(7):644-52. https://doi.org/10.1038/nbt.1883.
53. Haas BJ, Delcher AL, Mount SM, Wortman JR, Smith RK, Hannick LI, et al. Improving the Arabidopsis genome annotation using maximal transcript alignment assemblies. Nucleic Acids Res. 2003;31(19):5654-66. https://doi. org/10.1093/nar/gkg770.

54. Haas BJ, Salzberg SL, Zhu W, Pertea M, Allen JE, Orvis J, et al. Automated eukaryotic gene structure annotation using EVidenceModeler and the program to assemble spliced alignments. Genome Biol. 2008;9(1):R7. https:// doi.org/10.1186/gb-2008-9-1-r7.

55. Bairoch A, Apweiler R. The SWISS-PROT protein sequence database and its supplement TrEMBL in 2000. Nucleic Acids Res. 2000;28(1):45-8. https://doi. org/10.1093/nar/28.1.45

56. Zdobnov EM, Apweiler R. InterProScan-an integration platform for the signature-recognition methods in InterPro. Bioinformatics. 2001;17(9):847-8. https://doi.org/10.1093/bioinformatics/17.9.847.

57. Lowe TM, Eddy SR. tRNAscan-SE: a program for improved detection of transfer RNA genes in genomic sequence. Nucleic Acids Res. 1997;25(5): 955-64. https://doi.org/10.1093/nar/25.5.955.

58. Nawrocki EP, Kolbe DL, Eddy SR. Infernal 1.0: inference of RNA alignments. Bioinformatics. 2009;25(10):1335-7. https://doi.org/10.1093/bioinformatics/ btp157.

59. Griffiths-Jones S, Moxon S, Marshall M, Khanna A, Eddy SR, Bateman A. Rfam: annotating non-coding RNAs in complete genomes. Nucleic Acids Res. 2005;33(Database issue):D121-4. https://doi.org/10.1093/nar/gki081.

60. Krzywinski M, Schein J, Birol I, Connors J, Gascoyne R, Horsman D, et al. Circos: An information aesthetic for comparative genomics. Genome Res. 2009;19(9):1639-45. https://doi.org/10.1101/gr.092759.109.

61. Kim D, Landmead B, Salzberg SL. HISAT. a fast spliced aligner with low memory requirements. Nat Methods. 2015;12(4):357-60. https://doi.org/10.1 038/nmeth.3317.

62. Kent WJ. BLAT-The BLAST-like alignment tool. Genome Res. 2002;12(4):65664. https://doi.org/10.1101/gr.229202.

63. Li L, Stoeckert CJ, Roos DS. OrthoMCL: identification of ortholog groups for eukaryotic genomes. Genome Res. 2003;13(9):2178-89. https://doi.org/10.11 01/gr.1224503.

64. Yu XJ, Zheng HK, Wang J, Wang W, Su B. Detecting lineage-specific adaptive evolution of brain-expressed genes in human using rhesus macaque as outgroup. Genomics. 2006;88(6):745-51. https://doi.org/10.101 6/j.ygeno.2006.05.008.

65. Edgar RC. MUSCLE: multiple sequence alignment with high accuracy and high throughput. Nucleic Acids Res. 2004;32(5):1792-7. https://doi.org/10.1 093/nar/gkh340

66. Stamatakis A, Hoover P, Rougemont J. A rapid bootstrap algorithm for the RAxML web servers. Syst Biol. 2008;57(5):758-71. https://doi.org/10.1080/1 0635150802429642.

67. Yang ZH. PAML 4: phylogenetic analysis by maximum likelihood. Mol Biol Evol. 2007;24(8):1586-91. https://doi.org/10.1093/molbev/msm088.

68. Tang HB, Bowers JE, Wang XY, Ming R, Alam M, Paterson AH. Perspectivesynteny and collinearity in plant genomes. Science. 2008;320(5875):486-8. https://doi.org/10.1126/science.1153917.

69. De Bie T, Cristianini N, Demuth JP, Hahn MW. CAFE: a computational tool for the study of gene family evolution. Bioinformatics. 2006;22(10):1269-71. https://doi.org/10.1093/bioinformatics/btl097.

70. Zheng Y, Jiao C, Sun HH, Rosli HG, Pombo MA, Zhang PF, et al. iTAK: a program for genome-wide prediction and classification of plant transcription factors, transcriptional regulators, and protein kinases. Mol Plant. 2016;9(12):1667-70. https://doi.org/10.1016/j.molp.2016.09.014.

71. Slater GS, Birney E. Automated generation of heuristics for biological sequence comparison. BMC Bioinformatics. 2005;6(1):31. https://doi.org/1 0.1186/1471-2105-6-31.

72. Alexieva V, Sergiev I, Mapelli S, Karanov E. The effect of drought and ultraviolet radiation on growth and stress markers in pea and wheat. Plant Cell Environ. 2001;24(12):1337-44. https://doi.org/10.1046/j.1365-3040.2001.00778.x.

73. Bates LS, Waldren RP, Teare ID. Rapid determination of free proline for water-stress studies. Plant Soil. 1973;39(1):205-7. https://doi.org/10.1007/ BF00018060.

74. Li H, Handsaker B, Wysoker A, Fennell T, Ruan J, Homer N, et al. The sequence alignment/map format and SAMtools. Bioinformatics. 2009;25(16): 2078-9. https://doi.org/10.1093/bioinformatics/btp352.

75. Wang K, Li MY, Hakonarson H. ANNOVAR: functional annotation of genetic variants from high-throughput sequencing data. Nucleic Acids Res. 2010; 38(16):e164. https://doi.org/10.1093/nar/gkq603. 
76. Hudson RR, Slatkin M, Maddison WP. Estimation of levels of gene flow from DNA-sequence data. Genetics. 1992;132(2):583-9. https://doi.org/10.1093/ genetics/132.2.583.

77. Tajima F. Evolutionary relationship of DNA-sequences in finite populations. Genetics. 1983;105(2):437-60.

78. Ashburner M, Ball CA, Blake JA, Botstein D, Butler H, Cherry JM, et al. Gene Ontology: tool for the unification of biology. Nat Genet. 2000;25(1):25-9. https://doi.org/10.1038/75556.

79. Anders S, Pyl PT, Huber W. HTSeq-a Python framework to work with highthroughput sequencing data. Bioinformatics. 2015;31(2):166-9. https://doi. org/10.1093/bioinformatics/btu638.

80. Zhang JY, de Carvalho MHC, Torres-Jerez I, Kang Y, Allen SN, Huhman DV, et al. Global reprogramming of transcription and metabolism in Medicago truncatula during progressive drought and after rewatering. Plant Cell Environ. 2014:37(11):2553-76. https://doi.org/10.1111/pce.12328

81. Szklarczyk D, Gable AL, Lyon D, Junge A, Wyder S, Huerta-Cepas J, et al. STRING v11: protein-protein association networks with increased coverage, supporting functional discovery in genome-wide experimental datasets. Nucleic Acids Res. 2019;47(D1):D607-13. https://doi.org/10.1093/nar/gky1131.

82. Shannon P, Markiel A, Ozier O, Baliga NS, Wang JT, Ramage D, et al. Cytoscape. A software environment for integrated models of biomolecular interaction networks. Genome Res. 2003;13(11):2498-504. https://doi.org/1 $0.1101 /$ gr. 1239303

83. Higo K, Ugawa Y, Iwamoto M, Korenaga T. Plant cis-acting regulatory DNA elements (PLACE) database: 1999. Nucleic Acids Res. 1999;27:297-300.

84. Wang T. Medicago ruthenica. NCBI Genome WNNG00000000. 2021. https:// www.ncbinlm.nih.gov/genome/?term=WNNG00000000.

85. Institute of Botany, CAS. Medicago ruthenica RNA-seq reads. NCBI BioProject, PRJNA589938. 2019. https://www.ncbinlm.nih.gov/bioproject/PRJNA589938.

86. Institute of Botany, CAS. Medicago ruthenica re-sequencing reads. NCBI BioProject, PRJNA598783. 2020. https://www.ncbi.nlm.nih.gov/bioproject/ PRJNA598783.

87. Wang T. Genome assembly and annotation files of Medicago ruthenica. Figshare. 2021. https://doi.org/10.6084/m9.figshare.12726932.

\section{Publisher's Note}

Springer Nature remains neutral with regard to jurisdictional claims in published maps and institutional affiliations.

Ready to submit your research? Choose BMC and benefit from:

- fast, convenient online submission

- thorough peer review by experienced researchers in your field

- rapid publication on acceptance

- support for research data, including large and complex data types

- gold Open Access which fosters wider collaboration and increased citations

- maximum visibility for your research: over $100 \mathrm{M}$ website views per year

At $\mathrm{BMC}$, research is always in progress.

Learn more biomedcentral.com/submissions 